This item was submitted to Loughborough's Research Repository by the author.

Items in Figshare are protected by copyright, with all rights reserved, unless otherwise indicated.

\title{
Post- moderate intensity exercise energy replacement does not reduce subsequent appetite and energy intake in adolescents with obesity
}

\section{PLEASE CITE THE PUBLISHED VERSION}

https://doi.org/10.1017/S0007114519003106

\section{PUBLISHER}

Cambridge University Press (CUP)

\section{VERSION}

AM (Accepted Manuscript)

\section{PUBLISHER STATEMENT}

This article has been published in a revised form in British Journal of Nutrition https://doi.org/10.1017/S0007114519003106. This version is published under a Creative Commons CC-BYNC-ND. No commercial re-distribution or re-use allowed. Derivative works cannot be distributed. () The Authors.

\section{LICENCE}

CC BY-NC-ND 4.0

\section{REPOSITORY RECORD}

Thivel, David, Johanna Roche, Maud Miguet, Alicia Fillon, Marwa Khammassi, Kristine Beaulieu, Graham Finlayson, et al.. 2019. "Post- Moderate Intensity Exercise Energy Replacement Does Not Reduce Subsequent Appetite and Energy Intake in Adolescents with Obesity”. figshare. https://hdl.handle.net/2134/10676417.v1. 


\section{Post- moderate intensity exercise energy replacement does not reduce subsequent appetite and energy intake in adolescents with obesity}

Thivel $\mathrm{D}^{1,2,4}$, Roche $\mathrm{J}^{1}$, Miguet $\mathrm{M}$, Fillon $\mathrm{A}^{1,3}$, Khammassi $\mathrm{M}^{1}$, Beaulieu $\mathrm{K}^{4}$, Finlayson $\mathrm{G}^{4}$, Pereira $\mathrm{B}^{5}$, Miyashita $\mathrm{M}^{6}$, Thackray $\mathrm{AE}^{7}$, Masurier $\mathrm{J}^{3}$, Duclos $\mathrm{M}^{2,8,9,10}$, Boirie $\mathrm{Y}^{2,8,10,11}$

${ }^{1}$ Clermont Auvergne University, EA 3533, Laboratory of the Metabolic Adaptations to Exercise under Physiological and Pathological Conditions (AME2P), Clermont-Ferrand, France.

${ }^{2} \mathrm{CRNH}$-Auvergne, Clermont-Ferrand, France. ${ }^{3}$ UGECAM Nutrition Obesity Ambulatory Hospital, Clermont-Ferrand, France. ${ }^{4}$ School of Psychology, Faculty of Medicine and Health, University of Leeds, Leeds, LS2 9JT, UK ${ }^{5}$ Clermont-Ferrand University hospital, Biostatistics unit (DRCl), Clermont-Ferrand, France. ${ }^{6}$ Faculty of Sport Sciences, Waseda University, Saitama 169-8050, Japan.

${ }^{7}$ National Centre for Sport and Exercise Medicine, School of Sport, Exercise and Health Sciences, Loughborough University, Leicestershire LE11 3TU, UK.

8INRA, UMR 1019, Clermont-Ferrand, France.

${ }^{9}$ Department of Sport Medicine and Functional Explorations, Clermont-Ferrand University Hospital, G. Montpied Hospital, Clermont-Ferrand, France.

${ }^{10}$ University Clermont Auvergne, UFR Medicine, Clermont-Ferrand, France.

Running head: Post-exercise energy replacement and appetite

\section{Corresponding Author:}

Thivel David (PhD, HDR)

Clermont University, EA 3533, Laboratory of the Metabolic Adaptations to Exercise under Physiological and Pathological Conditions (AME2P), BP 80026, F-63171 Aubière cedex, France

David.thivel@uca.fr

Phone and fax/ 0033473407679

Conflict of Interest: No conflict of interest.

Funding: This study was funded by the French University Institute (IUF) and the UGECAM Nutrition Obesity Clinic.

Key words. Exercise; Energy Replacement; Appetite; Adolescents; Obesity 


\section{Abstract}

Exercise modifies energy intake in adolescents with obesity, but whether this is mediated by the exercise-induced energy deficit remains unknown. The present study examined the effect of exercise with and without dietary replacement of the exercise energy expenditure on appetite, energy intake and food reward in adolescents with obesity. Fourteen 12-15 years adolescents with obesity (8girls; Tanner3-4; BMI $34.8 \pm 5.7 \mathrm{~kg} / \mathrm{m}^{2}$; BMI-z score $2.3 \pm 0.4$ ) randomly completed 3 experimental conditions: i) rest control (CON); ii) 30-min cycling (EX); iii) 30-min cycling with dietary energy replacement $(E X+R)$. Ad libitum energy intake (EI) was assessed at lunch and dinner, and food reward (Leeds Food Preference Questionnaire) before and after lunch. Appetite was assessed at regular intervals. Lunch, evening and total El (excluding the post-exercise snack in EX-R) were similar across conditions. Lunch and total El including the post-exercise snack in EX+R were higher in EX-R than CON and EX; EX and CON were similar. Total relative El was lower in EX (1502 $\pm 488 \mathrm{kcal})$ compared with CON $(1713 \pm 530$; $\mathrm{p}<0.05)$ and higher in $E X+R(1849 \pm 486 \mathrm{kcal})$ compared with CON $(\mathrm{p}<0.001)$. Appetite and satiety quotients did not differ across conditions $(p \geq 0.10)$. Pre-meal explicit liking for fat was lower in $E X$ compared to $C O N$ and $E X+R(p=0.05)$. There was time by condition interaction between $E X$ and $C O N$ for explicit wanting and liking for fat $(p=0.01)$. Despite similar appetite and energy intake, adolescents with obesity do not adapt their post-exercise food intake to account for immediate dietary replacement of the exercise-induced energy deficit, favoring a short-term positive energy balance. 


\section{Introduction}

The prevalence of pediatric overweight and obesity represents a continuing global public health challenge ${ }^{(1)}$ and arises as a consequence of a chronic surplus of energy intake above energy expenditure ${ }^{(2)}$. Evidence supports a role for exercise in the control of body weight due to its ability to increase energy expenditure and induce a negative energy deficit in the absence of compensatory changes in energy intake ${ }^{(3)}$. The interplay between exercise, energy intake and appetite control in young people has attracted increasing scientific attention given the direct implications for energy homeostasis and body weight control.

It is well established that adults respond to acute moderate-to-high intensity exercise with a transient suppression in appetite and do not exhibit compensatory increases in appetite or ad libitum energy intake on the day of exercise ${ }^{(4 ; 5)}$. Single bouts of moderate-to-high intensity exercise have been shown to reduce ad libitum energy intake on the same day in children and adolescents with overweight or obesity but this effect has not been observed in healthy weight young people ${ }^{(6 ; 7 ; 8)}$. A recent review suggests that energy intake responses to acute exercise in young people may be modulated by physiological, neurocognitive and hedonic pathways ${ }^{(9)}$. Furthermore, it has been demonstrated recently that the exercise-induced reduction in ad libitum energy intake observed in adolescents with obesity appears to coincide with a reduction in food reward, evidenced by a reduction in the preference for high fat and sweet foods after exercise ${ }^{(10)}$. However, research examining hedonic responses to acute exercise stimuli in young people is sparse and further studies are required to enhance understanding of the interaction between exercise, appetite and energy intake in this population.

A handful of studies have examined the impact of energy intake manipulations in the immediate preor post-exercise periods on subsequent energy intake and appetite responses in young people. Specifically, it has been demonstrated that ingestion of a glucose solution immediately after 15 min of rest or moderate-to-vigorous intensity exercise reduced ad libitum energy intake independent of 
exercise in boys who were lean or overweight ${ }^{(11)}$. Similarly, a reduction in ad libitum energy intake has also been observed after a glucose preload was ingested before $40 \mathrm{~min}$ of rest or exercise in normal weight boys and men, but the effect was augmented when combined with the exercise bout (12). A recent study in adolescent boys and girls aged 12 to 14 years demonstrated that ad libitum energy intake was not altered in response to a mid-morning snack and an isoenergetic bout of cycling completed alone or in combination ${ }^{(13)}$. However, it is possible that the provision of a highly palatable pizza meal consumed in small peer groups may have influenced their ad libitum energy intake in the snack and exercise conditions ${ }^{(14)}$. The authors also reported that appetite was suppressed after snack intake but returned to control values before the ad libitum meal and were not influenced by exercise (13). However, it is not known whether replacement of the exercise-induced energy deficit immediately after exercise alters subsequent appetite, energy intake and food reward responses in adolescents with obesity.

Therefore, the aim of this study was to compare the effects of acute exercise with and without immediate replacement of the exercise-induced energy deficit on ad libitum energy intake, appetite perceptions and food reward in adolescents with obesity. We formulate the hypothesis that the adolescents will not reduce their ad libitum energy intake in presence of an energy replacement snack after exercise, favoring then a higher overall energy balance.

\section{Population}

Fourteen adolescents with obesity (according to Cole et al ${ }^{(15)}$ ) aged 12 to 15 years (Tanner stage 3-4) were recruited through the local Pediatric Obesity Centre (Tza Nou, La Bourboule, France) to participate in this study ( 6 boys, 8 girls). This study was conducted in accordance with the Declaration of Helsinki, approved by the local ethics authorities (Human Ethical Committee: CPP ILE DE FRANCE III; authorization reference: 2018-A02160-55) and registered with ClinicalTrials.gov (trial identifier: NCT03742622). All participants and their parent or legal guardian provided written informed assent or consent, respectively, before the study commenced. Participants were not taking any medications that could interact with the study outcomes, were engaging in less than $2 \mathrm{~h}$ of moderate physical 
activity per week (according to the IPAQ short-form questionnaire) ${ }^{(16)}$, and did not exhibit high cognitive restraint (as assessed using the Child Three-Factor Eating Questionnaire (CTFEQr17)) ${ }^{(17)}$.

\section{Preliminary measures}

After a preliminary medical screening visit with a clinical pediatrician to confirm eligibility, preliminary measurements were conducted to assess anthropometry and to determine peak oxygen uptake (peak $\mathrm{VO}_{2}$ ). Height and body mass were determined using a standard wall-mounted stadiometer and digital scale (SECA, Les Mureaux, France), respectively. Body Mass Index (BMI) was calculated as body mass $(\mathrm{kg})$ divided by height squared $\left(\mathrm{m}^{2}\right)$, and BMI percentile was calculated using age- and sex-specific French reference curves ${ }^{(18)}$. Fat mass (FM) and fat-free mass (FFM) were assessed by dual-energy X-ray absorptiometry (DXA) (QDR4500A scanner, Hologic, Waltham, MA, USA).

\section{Peak oxygen uptake $\left(\dot{V}_{2}\right)$ test}

Participants performed a peak $\dot{\mathrm{V}}_{2}$ test on a traditional concentric cycle ergometer ${ }^{(19)}$. The initial power was set at $30 \mathrm{~W}$ for 3 minutes, and was increased in $15 \mathrm{~W}$ increments every minute until volitional exhaustion. Maximal criteria were: heart rate $>90 \%$ of the age-predicted maximum heart rate $\left(210-0.65 \times\right.$ age), respiratory exchange ratio $(\mathrm{RER})>1.1$ and/or $\dot{\mathrm{VO}}_{2}$ plateau. Heart rate was monitored continuously using short-range telemetry (Polar V800, Polar Inc.) and 12-lead electrocardiography monitoring was conducted (Ultima SeriesTM, Saint Paul, MN). Oxygen consumption and carbon dioxide production were determined using an online breath-by-breath gas analysis system (BreezeSuite Software, Saint Paul, MN). Peak $\mathrm{VO}_{2}$ was defined as the average of the last $30 \mathrm{~s}$ of exercise before exhaustion.

\section{Main trials}

Participants completed 3, $12 \mathrm{~h}$ trials (08:00am-08:00 pm) in a random crossover design separated by one week: (1) rest control (CON); (2) exercise with energy deficit (EX); and (3) exercise with energy replacement $(E X+R)$. Participants arrived at the laboratory at 08:00am on the morning of the trials after a $12 \mathrm{~h}$ overnight fast. The adolescents were requested not to engage in any moderate-tovigorous physical activity during the two days that preceded each trial. Similarly, the adolescents were asked to avoid any food overconsumption and to record their intake on the day the preceded their first trial. They were asked to maintain a similar food consumption on the day before their two other trials.

During CON, participants were required to remain quiet and not to engage in any physical activity. During the exercise trials, participants cycled for $30 \mathrm{~min}$ (09:45am-10:15am) at $65 \%$ of their peak $\mathrm{V}_{2}$ and then rested in the laboratory. Heart rate was monitored continuously and the exercise-induced energy expenditure was estimated using data from the peak $\dot{\mathrm{V}}_{2}$ test. Immediately after the exercise session in EX-R, participants consumed an individually calibrated snack composed of bread, nuts, fruits and chocolate within 20 minutes to replace the estimated exercise-induced energy deficit (177 $\pm 39 \mathrm{kcal}$, respecting recommendations for age ${ }^{(20)}$. 
At 08:00am, participants consumed a standardised breakfast respecting the nutritional recommendation for age and consisting of white bread, butter, marmalade, yoghurt or semiskimmed milk and fruit or fruit juice which provided $500 \mathrm{kcal}$. Participants were provided with an ad libitum buffet meal for lunch (12:00 pm) and evening meal (07:00 pm). Lunch consisted of beef steak, pasta (Lustucru, Grenoble Isère; France), mustard (Auchan brand, Croix, France), cheese (Camembert Auchan brand, Croix, France), yoghurt (plain yoghurt, Auchan Brand, Croix, France), compote (apple compote Andros, Biars-sur-Cère, France), fruits and bread (white bread), and the evening meal consisted of ham or turkey, beans, mashed potato, cheese (Camembert Auchan brand, Croix, France), yoghurt (plain yoghurt, Auchan Brand, Croix, France), compote (apple compote Andros, Biars-sur-Cère, France), fruit and bread (white bread). Food items were provided in excess of expected consumption and participants were instructed to eat until "comfortably satiated". The adolescents made their choices and composed their trays individually. Their food selection was weighted by the investigators who served the adolescents. Importantly the adolescents were not aware that their plates were weighted and did not have any indication regarding the quantity of calories served. The weighted difference of food items was measured before and after the meal and intakes of energy and macronutrients were calculated using dietary analysis software (Bilnut v4.0 for Windows, La Fressinouze, France). Relative energy intake (REI) for the ad libitum lunch meal and total (ad libitum lunch and evening meals combined) were calculated as the energy intake minus the net energy expenditure of exercise.

\section{Subjective appetite ratings}

Appetite ratings were assessed throughout the day using visual analogue scales (150 millimeters scales) at baseline (fasted - 08:00 am), immediately after breakfast (08:30am), and then immediately before $(09: 45 \mathrm{am})$ and after $(10: 15 \mathrm{am})$ exercise, before $(12: 00 \mathrm{pm})$ and after $(01: 00 \mathrm{pm})$ lunch and before $(07: 00 \mathrm{pm})$ and after $(08: 00 \mathrm{pm})$ evening meal. Additional measurements were also obtained

19030 and 60 min after lunch ${ }^{(21)}$. Specifically, the questions i) "how hungry do you feel?", ii) "how full do you feel?", iii) "would you like to eat something?", and iv) "how much do you think you can eat?" provided an assessment of perceived hunger, fullness, drive to eat (DTE) and prospective food consumption (PFC), respectively. Total daily Area Under the Curves (AUC) for each appetite sensation were calculated as well as AUC for the 60 minutes post lunch. The satiety quotient (SQ) for hunger, fullness, PFC and DTE were calculated as follows ${ }^{(22)}$ :

Satiety quotient $(\mathrm{mm} / \mathrm{kcal})=\frac{\text { pre-lunch appetite }(\mathrm{mm})-\text { mean } 60 \text { min post-lunch appetite }(\mathrm{mm} / \mathrm{h})}{\text { energy content of lunch }(\mathrm{kcal})} \times 100$

\section{Food liking and wanting}

The Leeds Food Preference Questionnaire (LFPQ; described in detail by Dalton and Finlayson ${ }^{(23)}$ ) provided a measure of food preference and food reward. Participants were presented with an array of pictures of individual food items common in the diet. Foods in the array were chosen by the local research team from a validated database to be either predominantly high ( $>50 \%$ energy) or low $(<20 \%$ energy) in fat but similar in familiarity, protein content, palatability and suitable for the study 
population. The LFPQ has been deployed in a range of research studies ${ }^{(23)}$ including a recent exercise and appetite trial in young French boys ${ }^{(10)}$. Explicit liking and explicit wanting were measured by participants using $100 \mathrm{~mm}$ visual analogue scales to rate the extent they like each food ("How pleasant would it be to taste this food now?") and want each food ("How much do you want to eat this food now?"). The food images were presented individually, in a randomized order. Implicit wanting and relative food preference were assessed using a forced choice methodology in which the food images were paired so that every image from each of the four food types was compared to every other type over 96 trials (food pairs). Participants were instructed to respond as quickly and accurately as they could to indicate the food they wanted to eat the most at that time ("Which food do you most want to eat now?"). To measure implicit wanting, reaction times for all responses were covertly recorded and used to compute mean response times for each food type after adjusting for frequency of selection. Responses on the LFPQ were used to compute mean scores for high fat, low fat, sweet or savoury food types (and different fat-taste combinations). Fat bias scores were calculated as the difference between the high-fat scores and the low-fat scores, with positive values indicating greater liking, wanting or choice for high-fat relative to low-fat foods and negative values indicating greater liking, wanting or choice for low-fat relative to high-fat foods. Sweet bias scores were calculated as the difference between the sweet and savoury scores, with positive values indicating greater liking or wanting for sweet relative to savoury foods and negative values indicating greater liking or wanting for savoury relative to sweet foods.

\section{Statistical analyses}

Statistical analyses were performed using Stata software, Version 13 (StataCorp, College Station, TX, US). The sample size estimation was determined according to (i) CONSORT 2010 statement, extension to randomized pilot and feasibility trials (Eldridge et al., 2016) and (ii) Cohen's recommendations (Cohen, 1988) who has defined effect-size bounds as : small (ES: 0.2), medium (ES: 0.5 ) and large (ES: 0.8 , "grossly perceptible and therefore large"). So, with 12 patients by condition, an effect-size around 1 can be highlighted for a two-sided type I error at $1.7 \%$ (correction due to multiple comparisons), a statistical power greater than $80 \%$ and an intra-class correlation coefficient at 0.5 to take into account between and within participant variability. Continuous data was expressed as mean \pm standard deviation (SD) or median [interquartile range] according to statistical distribution. The assumption of normality was assessed using the Shapiro-Wilk test. Random-effects models for repeated data were performed with condition and time included as fixed factors and including a random effect for each participant. A Sidak's type I error correction was applied to perform multiple comparisons. As proposed by some statisticians ${ }^{24 ; 25)}$ a particular focus was also given to the magnitude of differences, in addition to inferential statistical tests expressed using $p$ values. The normality of residuals from these models was studied using the Shapiro-Wilk test. 
Although no gender difference was observed (certainly due to the relatively reduced sample size and then respective number of boys and girls), all statistical analyses were adjusted for gender.

\section{Results}

Fourteen $12.8 \pm 0.9$ years old adolescents with obesity participated in this study. Their mean body mass was $95.3 \pm 16.1 \mathrm{~kg}$, with a BMI of $34.8 \pm 5.7 \mathrm{~kg} / \mathrm{m}^{2}$ (z-BMI $\left.2.3 \pm 0.4\right)$, percentage of body fat mass of $37.7 \pm 4.2 \%$ and FFM of $57.4 \pm 8.2 \mathrm{~kg}$. The adolescents had a $\dot{\mathrm{VO}}_{2 \text { peak }}$ of $22.25 \pm 4.22$ $\mathrm{ml} / \mathrm{min} / \mathrm{kg}$. Energy expenditure induced by exercise (total duration $30 \mathrm{~min}$ ) was higher compared to the 30-min resting energy expenditure $(177 \pm 39 \mathrm{kcal}$ and $56 \pm 6 \mathrm{kcal}$, respectively; $p<0.001)$.

\section{Absolute and relative energy intake}

Table 1 displays the absolute and relative energy intake excluding the energy content of the postexercise snack in EX-R. Lunch, evening and total daily absolute ad libitum energy intakes were not different across the conditions (main effect of condition $P=0.09$ ).

Absolute lunch and total energy intake including the energy content of the post-exercise snack in EX$R$ were different across conditions (main effect of condition $p=0.008$ and $p=0.0013$, respectively)

255 (Figure 1A). Both lunch and total energy intake were higher in EX-R compared with CON and EX but no difference was seen between EX and CON (Figure 1A).

Lunch REI (including the post-exercise snack in EX-R; main effect of condition was higher in $E X+R$ $(1040 \pm 329 \mathrm{kcal})$ compared with both CON $(931 \pm 315 \mathrm{kcal})$ and EX ( $826 \pm 279 \mathrm{kcal})(p<0.001)$. Lunch REI had a tendency to be lower in EX versus CON ( $p=0.08)$. Total REI (including the post-exercise snack in EX-R; main effect of condition was lower in EX (1502 \pm 488 kcal) compared with CON (1713 \pm $530)(p<0.05)$ and higher in EX+R $(1849 \pm 486 \mathrm{kcal})$ compared with CON $(p<0.001)$. A tendency was found for total REI to be lower in EX compared with CON ( $p=0.07)$.

This Figure 2 illustrates the inter-individual variability of the lunch and total absolute and relative energy intake variations between the three experimental conditions (Figure 2).

\section{Macronutrient intake}

Absolute protein consumption at the evening meal was different across conditions (main effect of condition $p=)$, with intakes lower in EX (39.6 $\pm 16.9 \mathrm{~g}$ ) compared with CON (58.6 $\pm 25.8 \mathrm{~g}, \mathrm{p}=0.009$ ) and $E X+R(51.1 \pm 16.8 \mathrm{~g}, \mathrm{p}=0.0253)$. No differences were observed in absolute protein intake at lunch or overall, or in absolute carbohydrate and fat intake at lunch, evening meal or overall.

The percentage of energy ingested from protein and $\mathrm{CHO}$ was not different across conditions at lunch, evening meal or overall. At the evening meal, the percentage of energy ingested through fat was lower in CON $(21.9 \pm 10.8 \%)$ compared with both EX $(31.3 \pm 9.7 \%, p=0.043)$ and EX+R $(32.1 \pm$ $7.8 \%, p=0.023$ ). In total (lunch and evening meal combined), the percentage of energy ingested from 

marginally higher in EX-R than EX $(27.5 \pm 3.8 \%)(p=0.063)$.

\section{Appetite ratings}

As detailed in Table 2, none of the fasting, pre-lunch or total AUC values for hunger, fullness, PFC and DTE were different across conditions (all main effects of condition $p \geq 0.10$ ). The AUC 60 minutes post lunch and satiety quotients, as satiating indicators, did not differ across conditions for any of the appetite ratings (all main effects of condition $\mathrm{P}=0.11$ ).

\section{Food reward}

As detailed in Table 3, no condition (exercise versus control), time (pre versus post meal) or interaction (time $\mathrm{x}$ condition) effects were found for Choice Taste Bias, Implicit Wanting Taste and Fat Bias, and Explicit Liking Taste Bias. Choice Fat Bias did not show any condition or interaction effect. Explicit Wanting Fat Bias was significantly reduced in response to the test meal in CON only $(p=0.001)$ and a time $x$ condition interaction was observed between CON and EX ( $p=0.017)$. Explicit Wanting Taste Bias only showed a significant reduction in response to the ad libitum test meal in CON ( $p=0.03)$. Pre-meal Explicit Liking Fat Bias showed a significant condition effect $(p=0.05)$ with EX being significantly lower than both $\operatorname{CON}(p=0.016)$ and $E X+R(p=0.01)$. Explicit Liking Fat Bias also showed a condition $x$ time interaction between CON and EX ( $p=0.026)$.

\section{Discussion}

In accordance with our initial hypothesis, the primary finding of the present study was that 30minutes of moderate-intensity cycling did not alter subsequent ad libitum energy intake or appetite in adolescents with obesity, irrespective of whether the exercise-induced energy deficit was replaced immediately after exercise. The absence of adjustments in post-exercise energy intake suggests that maintenance of the exercise-induced energy deficit may be required to prevent the promotion of a positive energy balance on the same day of exercise.

Absolute energy intake at the ad libitum lunch and evening meals were similar between conditions resulting in higher energy intake in EX+R when accounting for the energy content of the post- 
exercise snack. This resulted in a lower REI when the energy deficit was maintained after exercise, but REI was higher in EX+R than CON indicative of a positive energy balance on the day of exercise when the exercise-induced energy deficit was replaced. The lack of modification in post-exercise absolute energy intake might be explained by the moderate intensity of the exercise bout. Indeed, while our results are in line with some studies using similar exercise intensities ${ }^{(26 ;}{ }^{27)}$, other studies conducted in similar populations reported reduced energy intake after single bouts of vigorousintensity exercise (>70\% of maximal capacity) ${ }^{(10 ; 28 ; 29)}$. This anorexigenic effect of vigorous-intensity exercise in adolescents with obesity has been confirmed in a recent systematic review and metaanalysis ${ }^{(8)}$. It is also possible that the absence of energy intake modification after exercise with an energy deficit in the present study may reflect the delay between the exercise bout and the ad libitum lunch meal. Previous studies have typically provided an ad libitum test meal approximately 30 minutes after exercise cessation ${ }^{26 ; 29)}$ whereas the ad libitum lunch meal was provided $2 \mathrm{~h}$ after exercise in the current investigation. In this regard, Albert et al. reported lower food intake after moderate-intensity exercise completed 30 minutes, but not 130 minutes, before a test meal in healthy weight adolescent boys ${ }^{(30)}$. Our study was not designed to investigate the importance of energy intake timing in the post-exercise period; therefore, further research is required before recommendations can be made.

The effect of immediate replacement of the exercise-evoked energy expenditure on subsequent energy intake responses in young people is restricted to one previous investigation in 12 to 14 yearold adolescents ${ }^{(13)}$. In accord with our findings, Varley-Campbell et al. did not find any differences in energy intake after acute exercise regardless of whether the exercise energy expenditure was replaced, resulting in a lower relative energy intake when the energy deficit was maintained ${ }^{(13)}$. Importantly, the authors employed a pizza buffet meal, which may have reduced the sensitivity to detect differences in energy intake due to the high palatability of the meal ${ }^{(14)}$. Consequently, the present work adopted a balanced buffet meal that was designed to provide familiar foods without promoting over-, under- or occasional/opportunistic consumption (as previously validated ${ }^{(31)}$ ). 
Furthermore, the buffet meals adopted in this study allowed the exploration of specific macronutrient intakes. While we did not find any modification in the absolute consumption of fats, proteins and carbohydrates at lunch, absolute protein intake was lower at the evening meal after EX but not after EX+R. Interestingly, the total daily percentage of energy derived from fat was higher in EX+R compared to CON and EX. The reason underpinning these findings is unclear, with previous evidence examining the effect of exercise on macronutrient intake in young people yielded largely conflicting findings ${ }^{(8)}$. Nevertheless, the findings of the present study contribute to the extant literature examining energy and macronutrient intake responses to acute exercise in adolescents.

It has been shown previously in healthy weight boys and girls that ingestion of a mid-morning snack, both before acute exercise and an equivalent period or rest, suppressed hunger and PFC and elevated fullness compared to exercise and rest conditions with no snack provision ${ }^{(13)}$. The present study in adolescents with obesity observed no differences in any of the fasting, pre-meal or total daily appetite sensations after exercise inducing an energy deficit, supporting previous studies in adolescents with obesity ${ }^{(26 ; 32)}$. Our findings extend the current evidence base by demonstrating that subjective appetite is not altered in adolescents with obesity when the energy expenditure induced by exercise is replaced, contrasting the previously discussed findings in healthy weight boys and girls ${ }^{(13)}$. Clearly, the effect of acute exercise on appetite ratings and the interplay between subjective appetite sensations and energy intake requires further attention in this population, particularly given the largely contradictory results evident in the present literature (for review see ${ }^{(33)}$ ).

The Leeds Food Preference Questionnaire was adopted in this study to assess the adolescents' food reward immediately before and in response to an ad libitum lunch meal. Evidence examining the effect of acute exercise on food reward are relatively sparse ${ }^{(34 ; 35 ; 36)}$, especially in children and adolescents. Nevertheless, it has been reported recently that the preference for high-fat vs. low-fat foods was reduced in response to single bouts of aerobic and resistance exercise in healthy adult women using the LFPQ ${ }^{(36)}$. Furthermore, the hedonic "liking" of high-fat foods was lower after 
resistance, but not after aerobic exercise, suggesting a potential role for exercise modality ${ }^{(36)}$. In adolescents with obesity, Miguet and colleagues showed recently that 16-minutes of high-intensity interval cycling decreased the relative preference for both fat and sweet taste, and implicit wanting for high-fat foods (using the LFPQ) in response to an ad libitum meal ${ }^{(10)}$. The present results also seem to suggest a potential effect of acute exercise on food reward in this population with a reduced pre-meal explicit liking for fat compared to the control condition which was not altered in the presence of post-exercise energy replacement $(E X+R)$. Explicit Wanting for fat showed a significant interaction between CON and EX with a lower reduction in response to the test meal on EX compared with CON, whereas no time $x$ condition interaction was observed between CON and EX+R. A significant interaction was also observed between CON and EX for Explicit Liking for fat that increased in response to the test meal in EX and decreased in CON. This suggests that an exerciseinduced energy deficit may influence food reward in adolescents with obesity, while these effects are diminished after immediate replacement of the energy expenditure induced by exercise. Further work is required to confirm these findings and to determine the relevance in relation to exercise, appetite and energy balance in young people.

The present study is limited by the absence of a direct measurement of energy expenditure during the exercise bouts which may have under- or over-estimated the exercise-induced energy deficit. Longer exercise and/or performed at higher intensities might be considered to favor greater energy deficits, which might lead to divergent results. Other limitations like the absence of hormonal 375 indicators (mainly those involved in the control of energy intake), the lack of control of the level of hydration of the adolescents and the relatively reduced sample size have to be considered when interpreting our results. Nevertheless, this study is the first to investigate the effect of post-exercise energy replacement on the subsequent appetite and energy intake responses in adolescents with obesity, a population where weight control strategies are likely to provoke the most clinical relevance. 
In conclusion, adolescents with obesity do not alter their post-exercise ad libitum energy intake after immediate dietary replacement of the exercise-induced energy deficit. This results in a short-term positive energy balance which, if sustained over the long term, may have important implications for weight control in this population. Further work is required to confirm the clinical relevance of these findings, but cautious adoption of post-exercise energy replacement practices may be required in adolescents with obesity to optimize the beneficial effects of exercise.

\section{Authors' contribution}

TD, MJ, DM, TA and YB designed the study. RJ, MM, FA, KM performed the data collection. KB, MM, GF, TD PB analyzed and interpreted the data. TD, YB, DM, AT and MM wrote and revised the paper.

\section{References}

1. (2017) Worldwide trends in body-mass index, underweight, overweight, and obesity from 1975 to 2016: a pooled analysis of 2416 population-based measurement studies in 128.9 million children, adolescents, and adults. Lancet 390, 2627-2642.

395 2. Swinburn BA, Sacks G, Hall KD et al. (2011) The global obesity pandemic: shaped by global drivers and local environments. Lancet 378, 804-814.

3. Donnelly JE, Blair SN, Jakicic JM et al. (2009) American College of Sports Medicine Position Stand. Appropriate physical activity intervention strategies for weight loss and prevention of weight regain for adults. Med Sci Sports Exerc 41, 459-471.

400 4. Deighton K, Stensel DJ (2014) Creating an acute energy deficit without stimulating compensatory increases in appetite: is there an optimal exercise protocol? Proc Nutr Soc 73, 352-358.

5. Schubert MM, Desbrow B, Sabapathy $S$ et al. (2013) Acute exercise and subsequent energy intake. A meta-analysis. Appetite 63, 92-104.

6. Thivel D, Metz L, Julien A et al. (2014) Obese but not lean adolescents spontaneously decrease energy intake after intensive exercise. Physiol Behav 123, 41-46.

7. Fearnbach SN, Silvert L, Pereira B et al. (2017) Reduced neural responses to food cues might contribute to the anorexigenic effect of acute exercise observed in obese but not lean adolescents. Nutr Res 44, 76-84.

8. Thivel D, Rumbold PL, King NA et al. (2016) Acute post-exercise energy and macronutrient intake in lean and obese youth: a systematic review and meta-analysis. Int J Obes 40, 1469-1479.

9. Thivel D, Finlayson G, Blundell JE (2019) Homeostatic and neurocognitive control of energy intake in response to exercise in pediatric obesity: a psychobiological framework. Obes Rev 20, 316-324.

10. Miguet $M$, Fillon A, Khammassi $M$ et al. (2018) Appetite, energy intake and food reward responses to an acute High Intensity Interval Exercise in adolescents with obesity. Physiol Behav 195, 90-97.

11. Tamam S, Bellissimo N, Patel BP et al. (2012) Overweight and obese boys reduce food intake in response to a glucose drink but fail to increase intake in response to exercise of short duration. Appl Physiol Nutr Metab 37, 520-529.

12. Hunschede S, Kubant R, Akilen R et al. (2017) The Role of IL-6 in Appetite Suppression after HighIntensity Exercise in Normal-weight and Obese Boys. Current Developments in Nutrition 1, e000398. 
13. Varley-Campbell JL, Moore MS, Williams CA (2017) The effects of a mid-morning snack and moderate-intensity exercise on acute appetite and energy intake in 12-14-year-old adolescents. $\mathrm{Br} \mathrm{J}$ Nutr 117, 602-610.

14. Thivel D, Duché P, Morio B (2012) Discussion of "overweight and obese boys reduce food intake in response to a glucose drink but fail to increase intake in response to exercise of short duration". Appl Physiol Nutr Metab 37, 1014-1015; discussion 1016-1017.

15. Cole TJ, Bellizzi MC, Flegal KM et al. (2000) Establishing a standard definition for child overweight and obesity worldwide: international survey. BMJ 320, 1240-1243.

16. Craig CL, Marshall AL, Sjostrom M et al. (2003) International physical activity questionnaire: 12country reliability and validity. Med Sci Sports Exerc 35, 1381-1395.

17. Bryant EJ, Thivel D, Chaput JP et al. (2018) Development and validation of the Child Three-Factor Eating Questionnaire (CTFEQr17). Public Health Nutr 21, 2558-2567.

18. (2006) WHO Child Growth Standards based on length/height, weight and age. Acta Paediatr Suppl 450, 76-85.

435 19. Rowland TW (1996) Developmental Exercise Physiology. Human Kinetics ed ed. Champaign, IL.

20. Murphy SP, Poos MI (2002) Dietary Reference Intakes: summary of applications in dietary assessment. Public Health Nutr 5, 843-849.

21. Flint A, Raben A, Blundell JE et al. (2000) Reproducibility, power and validity of visual analogue scales in assessment of appetite sensations in single test meal studies. Int J Obes Relat Metab Disord

$440 \quad 24,38-48$.

22. Drapeau V, King N, Hetherington M et al. (2007) Appetite sensations and satiety quotient: predictors of energy intake and weight loss. Appetite 48, 159-166.

23. Dalton M, Finlayson G (2014) Psychobiological examination of liking and wanting for fat and sweet taste in trait binge eating females. Physiol Behav 136, 128-134.

445 24. Feise RJ (2002) Do multiple outcome measures require p-value adjustment? BMC Med Res Methodol 2, 8.

25. Rothman, Greenland (1998) Modern epidemiology. 2nd ed. Philadelphia, PA: Lippencott-Raven Publishers.

26. Masurier J, Mathieu ME, Fearnbach SN et al. (2018) Effect of exercise duration on subsequent appetite and energy intake in obese adolescent girls. Int I Sport Nutr Exerc Metab 28, 593-601.

27. Thivel D, Doucet $E$, Julian V et al. (2017) Nutritional compensation to exercise- vs. diet-induced acute energy deficit in adolescents with obesity. Physiol Behav 176, 159-164.

28. Prado WL, Balagopal PB, Lofrano-Prado MC et al. (2015) Effect of aerobic exercise on hunger feelings and satiety regulating hormones in obese teenage girls. Pediatr Exerc Sci 26, 463-469.

455 29. Thivel D, Isacco L, Montaurier C et al. (2012) The 24-h Energy Intake of Obese Adolescents Is Spontaneously Reduced after Intensive Exercise: A Randomized Controlled Trial in Calorimetric Chambers. PLoS One 7, e29840.

30. Albert MH, Drapeau V, Mathieu ME (2015) Timing of moderate-to-vigorous exercise and its impact on subsequent energy intake in young males. Physiol Behav 151, 557-562.

460 31. Thivel D, Genin PM, Mathieu ME et al. (2016) Reproducibility of an in-laboratory test meal to assess ad libitum energy intake in adolescents with obesity. Appetite 105, 129-133.

32. Fearnbach SN, Silvert L, Keller KL et al. (2016) Reduced neural response to food cues following exercise is accompanied by decreased energy intake in obese adolescents. Int J Obes (Lond) 40, 7783.

465 33. Thivel D, Chaput JP (2014) Are post-exercise appetite sensations and energy intake coupled in children and adolescents? Sports Med 44, 735-741.

34. Finlayson G, Bryant E, Blundell JE et al. (2009) Acute compensatory eating following exercise is associated with implicit hedonic wanting for food. Physiol Behav 97, 62-67.

35. Farah NM, Brunstrom JM, Gill JM (2012) Using a novel computer-based approach to assess the

470 acute effects of exercise on appetite-related measures. Appetite 58, 196-204.

36. McNeil J, Cadieux S, Finlayson G et al. (2015) The effects of a single bout of aerobic or resistance exercise on food reward. Appetite 84, 264-270. 


\section{Figures' list}

475 Figure 1. Absolute (A) and relative (B) energy intake in the control (CON), exercise with energy deficit $(E X)$ and exercise with energy replacement $(E X+R)$ conditions. Values are mean (SD) for $n=14$. Values for EX-R include the energy content of the post-exercise snack. ( $* * 0<0.05$ for the main effect of condition; a: $p<0.05$ versus control; $c$ : $p<0.001$ versus Exercise).

Figure 2. Individual variation of absolute energy intake at lunch (A) and total (B) and of relative energy intake at lunch (C) and total (D) in the control (CON), exercise with energy deficit (EX) and exercise with energy replacement $(E X+R)$ conditions. Values are mean $(S D)$ for $n=14$. Values for $E X-R$ include the energy content of the post-exercise snack. 


\section{Tables}

Table 1. Absolute and relative ad libitum energy intake in the control, exercise with energy deficit (EX) and exercise with energy replacement (EX+R) conditions.

\begin{tabular}{|c|c|c|c|c|c|c|c|}
\hline & \multicolumn{2}{|c|}{ CON } & \multicolumn{2}{|c|}{ EX } & \multicolumn{2}{|c|}{ EX+R } & \multirow{2}{*}{$\mathbf{P}$} \\
\hline & Mean & SD & Mean & SD & Mean & SD & \\
\hline \multicolumn{8}{|c|}{ Absolute Energy Intake (kcal) } \\
\hline Lunch & 987 & 315 & 1003 & 289 & 1040 & 329 & 0.44 \\
\hline Evening & 782 & 319 & 676 & 295 & 809 & 177 & 0.17 \\
\hline Total & 1769 & 532 & 1678 & 501 & 1849 & 486 & 0.09 \\
\hline \multicolumn{8}{|c|}{ Relative Energy Intake (kcal) } \\
\hline Lunch & 931 & 315 & 826 & 279 & 1040 & $329 *, c$ & 0.0032 \\
\hline Total & 1713 & 530 & 1502 & $488^{*}$ & 1849 & $486^{\mathrm{C}}$ & 0.0020 \\
\hline
\end{tabular}

Values are mean (SD) for $n=14$ and are exclusive of the post-exercise snack consumed in EX-R. ${ }^{*} p<0.05$ versus CON.

${ }^{C_{p}<0.001}$ versus EX; $P$ values represent the main effect of condition. 
Table 2. Fasting, total area under the curve and satiety quotients for each appetite rating in the control (CON), exercise with energy deficit (EX) and exercise with energy replacement $(E X+R)$ conditions.

\begin{tabular}{|c|c|c|c|c|c|c|c|}
\hline & \multicolumn{2}{|c|}{ CON } & \multicolumn{2}{|c|}{ EX } & \multicolumn{2}{|c|}{$E X+R$} & \multirow{2}{*}{$\mathbf{P}$} \\
\hline & Mean & SD & Mean & SD & Mean & SD & \\
\hline \multicolumn{8}{|l|}{ Hunger } \\
\hline Fasting (mm) & 68 & 48 & 81 & 48 & 97 & 38 & 0.10 \\
\hline Pre-lunch (mm) & 91 & 36 & 83 & 45 & 87 & 37 & 0.76 \\
\hline $\mathrm{SQ}$ (mm/kcal) & 8,7 & 3,5 & 7,5 & 4,3 & 8,1 & 5,1 & 0.59 \\
\hline AUC $60 \mathrm{~min}$ post lunch $(\mathrm{mm})$ & 570 & 681 & 633 & 997 & 665 & 1061 & 0.82 \\
\hline Total AUC (mm) & 9243 & 3747 & 9526 & 4703 & 9841 & 4304 & 0.51 \\
\hline \multicolumn{8}{|l|}{ Fullness } \\
\hline Fasting (mm) & 33 & 55 & 21 & 33 & 9 & 9 & 0.20 \\
\hline Pre lunch meal (mm) & 22 & 25 & 27 & 40 & 23 & 30 & 0.97 \\
\hline $\mathrm{SQ}$ (mm/kcal) & $-10,1$ & 4,6 & $-5,4$ & 9,8 & $-8,1$ & 5,2 & 0.26 \\
\hline AUC $60 \mathrm{~min}$ post lunch $(\mathrm{mm})$ & 7139 & 2037 & 4953 & 3442 & 5987 & 2746 & 0.11 \\
\hline Total AUC (mm) & 23180 & 6988 & 17837 & 8877 & 19881 & 7918 & 0.17 \\
\hline \multicolumn{8}{|l|}{ PFC } \\
\hline Fasting (mm) & 71 & 43 & 80 & 44 & 85 & 40 & 0.69 \\
\hline Pre lunch meal (mm) & 97 & 26 & 94 & 42 & 89 & 38 & 0.82 \\
\hline $\mathrm{SQ}$ (mm/kcal) & 9,1 & 3,8 & 8,6 & 5,9 & 7,6 & 4,3 & 0.62 \\
\hline AUC $60 \mathrm{~min}$ post lunch $(\mathrm{mm})$ & 888 & 1141 & 869 & 1095 & 905 & 1267 & 0.94 \\
\hline Total AUC (mm) & 10253 & 5005 & 11297 & 5106 & 10574 & 5444 & 0.84 \\
\hline \multicolumn{8}{|l|}{ DTE } \\
\hline Fasting $(\mathrm{mm})$ & 74 & 48 & 87 & 48 & 96 & 42 & 0.30 \\
\hline Pre lunch meal (mm) & 100 & 36 & 96 & 44 & 89 & 39 & 0.63 \\
\hline $\mathrm{SQ}$ (mm/kcal) & 9,6 & 3,9 & 8,3 & 3,8 & 7,8 & 4,6 & 0.31 \\
\hline AUC $60 \mathrm{~min}$ post lunch $(\mathrm{mm})$ & 659 & 960 & 662 & 777 & 763 & 1145 & 0.79 \\
\hline Total AUC (mm) & 10576 & 4922 & 11106 & 6076 & 9701 & 4826 & 0.42 \\
\hline
\end{tabular}

495 Values are mean (SD) for $\mathbf{n}=14$. SQ: Satiety Quotient; DTE: Desire To Eat; PFC: Prospective Food Consumption; AUC; Area Under the Curve; SD: Standard Deviation. P values represent the main effect of condition. 
505 Table 3. Pre- and post-lunch meal food reward in the control (CON), exercise with energy deficit (EX) and exercise with energy replacement (EX-R) conditions.

\begin{tabular}{|c|c|c|c|c|c|c|c|c|c|c|}
\hline & \multicolumn{2}{|c|}{ CON } & \multicolumn{2}{|c|}{ EX } & \multicolumn{2}{|c|}{$E X+R$} & \multirow[b]{2}{*}{$\mathbf{p}$} & \multicolumn{3}{|c|}{ Interaction time $\mathrm{x}$ condition } \\
\hline & Mean & SD & Mean & SD & Mean & SD & & CON vs. EX & CON vs. EX+R & $E X$ vs. $E X+R$ \\
\hline \multicolumn{11}{|l|}{ Choice } \\
\hline \multicolumn{11}{|l|}{ Fat Bias } \\
\hline Before meal & 6,4 & 10,4 & 5,6 & 10,0 & 4,8 & 7,2 & 0,71 & \multirow{2}{*}{0,97} & \multirow{2}{*}{0,76} & \multirow{2}{*}{0,75} \\
\hline After meal & 8,29 & 10,1 & 7,6 & 8,8 & 6,5 & 6,3 & 0,42 & & & \\
\hline$p$ before vs. after meal & \multicolumn{2}{|c|}{0,01} & \multicolumn{2}{|c|}{0,22} & \multicolumn{2}{|c|}{0,26} & & & & \\
\hline \multicolumn{11}{|l|}{ Taste Bias } \\
\hline Before meal & 5,5 & 12,3 & 2,15 & 13,0 & 4,4 & 11,8 & 0,49 & \multirow{3}{*}{0,21} & \multirow{3}{*}{0,35} & \multirow{3}{*}{0,74} \\
\hline After meal & 5,7 & 12,4 & 5,54 & 11,8 & 7,4 & 13,2 & 0,68 & & & \\
\hline$p$ before vs. after meal & \multicolumn{2}{|c|}{0,88} & \multicolumn{2}{|c|}{0,11} & \multicolumn{2}{|c|}{0,06} & & & & \\
\hline \multicolumn{11}{|l|}{ Implicit Wanting } \\
\hline \multicolumn{11}{|l|}{ Fat Bias } \\
\hline Before meal & 4,6 & 40,3 & 16,29 & 25,9 & 2,6 & 27,9 & 0,22 & \multirow{3}{*}{0,06} & \multirow{2}{*}{0,24} & \multirow{3}{*}{0,16} \\
\hline After meal & 28,3 & 47,1 & 12,51 & 13,7 & 24,4 & 82,9 & 0,18 & & & \\
\hline$p$ before vs. after meal & 0, & & 0,2 & & 0, & & & & & \\
\hline Taste Bias & & & & & & & & & & \\
\hline Before meal & 18,5 & 39,8 & $-0,26$ & 42,0 & 15,5 & 30,3 & 0,78 & 0.57 & 0.99 & 032 \\
\hline After meal & 20,7 & 34,6 & 17,51 & 29,4 & 18,7 & 36,1 & 0,73 & וכנ, & ככ, & (כ) \\
\hline$p$ before vs. after meal & 0,5 & & 0,1 & & 0, & & & & & \\
\hline Explicit Wanting & & & & & & & & & & \\
\hline Fat Bias & & & & & & & & & & \\
\hline Before meal & 15,5 & 7,5 & 11,90 & 7,5 & 15,1 & 14,7 & 0,80 & 0017 & 084 & \\
\hline After meal & 4,7 & 6,1 & 10,74 & 9,0 & 5,7 & 10,4 & 0,32 & ו & 0,04 & 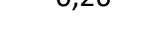 \\
\hline$p$ before vs. after meal & 0,0 & & 0,5 & & 0, & & & & & \\
\hline Taste Bias & & & & & & & & & & \\
\hline Before meal & 17,2 & 12,2 & 16,10 & 12,0 & 17,6 & 20,8 & 0,87 & 0.94 & 078 & 084 \\
\hline After meal & 9,3 & 9,4 & 8,60 & 10,6 & 11,8 & 11,6 & 0,64 & & & \\
\hline$p$ before vs. after meal & 0,0 & & 0,1 & & 0, & & & & & \\
\hline Explicit Liking & & & & & & & & & & \\
\hline Fat Bias & & & & & & & & & & \\
\hline Before meal & 14,4 & $7,4^{* *}$ & 1,60 & 12,9 & 9,0 & $16,4^{* *}$ & 0,05 & 0.026 & 0.50 & 0.25 \\
\hline After meal & 7,9 & 9,7 & 8,08 & 8,2 & 7,3 & 8,1 & 0,97 & $0, v<0$ & ט, & \\
\hline$p$ before vs. after meal & 0,0 & & 0,2 & & 0, & & & & & \\
\hline Taste Bias & & & & & & & & & & \\
\hline Before meal & 17,7 & 12,0 & 8,90 & 18,1 & 15,0 & 18,5 & 0,23 & 0,22 & 0,17 & 0,76 \\
\hline After meal & 9,2 & 10,3 & 7,46 & 9,4 & 18,6 & 15,9 & 0,15 & & 0,11 & 0,10 \\
\hline$p$ before vs. after meal & 0,0 & & 0,5 & & 0, & & & & & \\
\hline
\end{tabular}

Values are mean (SD) for $n=14$. CON: control condition; EX: Exercise condition; EX+R: Exercise + Energy Replacement condition; SD: Standard Deviation, ${ }^{* *} \mathrm{p}<0.01$ versus EX 
Figure 1

A

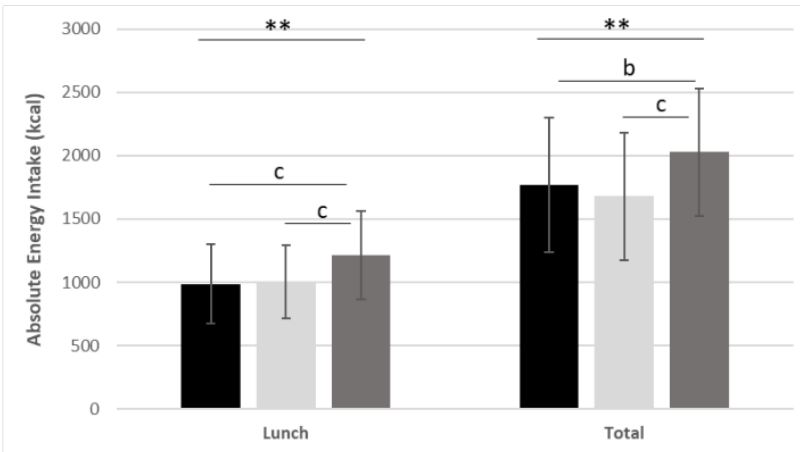

B

EX

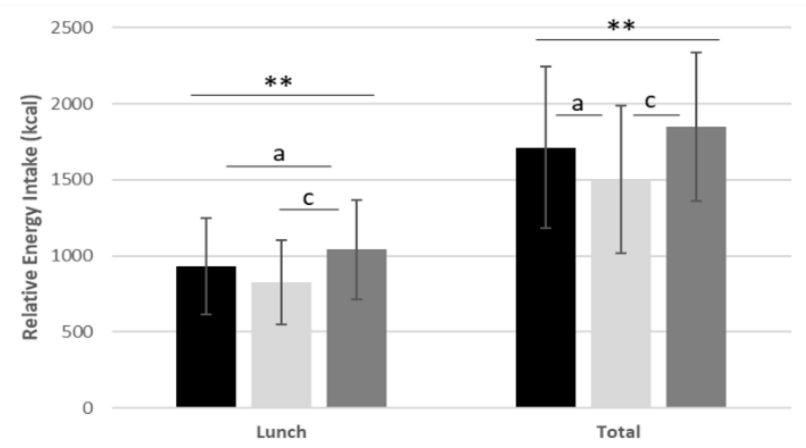




\section{Figure 2}
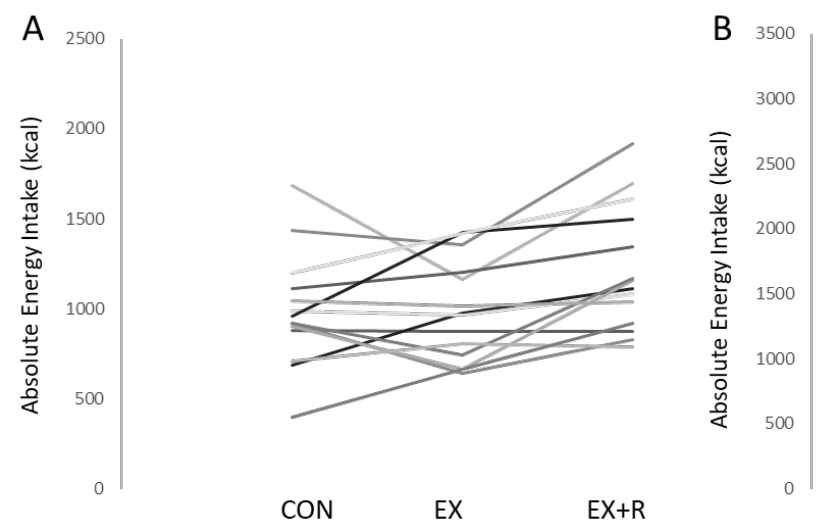

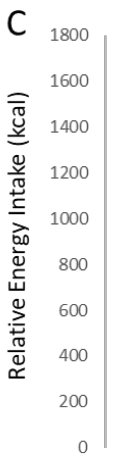

Lunch

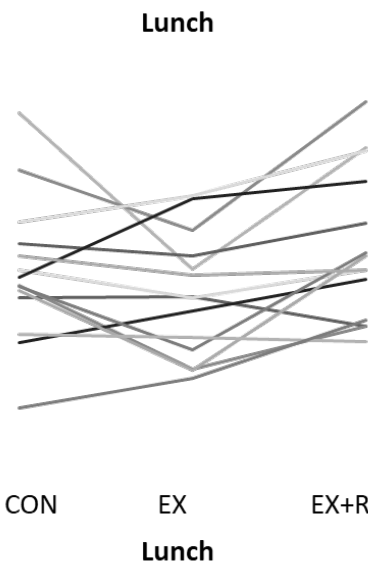

$D_{3000}$

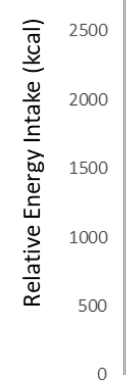

CON EX $\quad E X+R$

Total

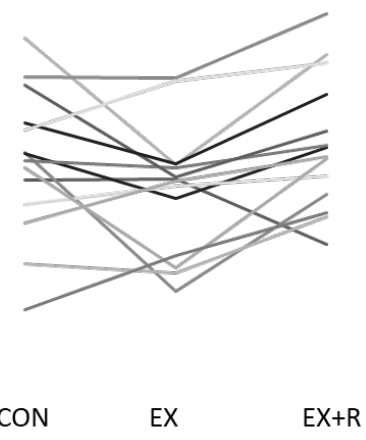

Total 